\title{
Video Article \\ Carrier Lifetime Measurements in Semiconductors through the Microwave Photoconductivity Decay Method
}

\author{
Takato Asada ${ }^{1}$, Yoshihito Ichikawa ${ }^{1}$, Masashi Kato ${ }^{1,2}$ \\ ${ }^{1}$ Department of Electrical \& Mechanical Engineering, Nagoya Institute of Technology \\ ${ }^{2}$ Frontier Research Institute for Material Science, Nagoya Institute of Technology
}

Correspondence to: Masashi Kato at kato.masashi@nitech.ac.jp

URL: https://www.jove.com/video/59007

DOI: doi:10.3791/59007

Keywords: Engineering, Issue 146, microwave photoconductivity decay method, career lifetime, semiconductor material, time resolved measurement, defect characterization, surface recombination

Date Published: 4/18/2019

Citation: Asada, T., Ichikawa, Y., Kato, M. Carrier Lifetime Measurements in Semiconductors through the Microwave Photoconductivity Decay Method. J. Vis. Exp. (146), e59007, doi:10.3791/59007 (2019).

\section{Abstract}

This work presents a protocol employing the microwave photoconductivity decay ( $\mu$-PCD) for measurement of the carrier lifetime in semiconductor materials, especially $\mathrm{SiC}$. In principle, excess carriers in the semiconductor generated via excitation recombine with time and, subsequently, return to the equilibrium state. The time constant of this recombination is known as the carrier lifetime, an important parameter in semiconductor materials and devices that requires a noncontact and nondestructive measurement ideally achieved by the $\mu$-PCD. During irradiation of a sample, a part of the microwave is reflected by the semiconductor sample. Microwave reflectance depends on the sample conductivity, which is attributed to the carriers. Therefore, the time decay of excess carriers can be observed through detection of the reflected microwave intensity, whose decay curve can be analyzed for estimation of the carrier lifetime. Results confirm the suitability of the $\mu-P C D$ protocol in measuring the carrier lifetime in semiconductor materials and devices.

\section{Video Link}

The video component of this article can be found at https://www.jove.com/video/59007/

\section{Introduction}

Excess carriers in semiconductors are optically excited by the injection of photons with energy larger than the gap between the conduction and valence bands. Excited excess carriers, then, disappear by an electron-hole recombination within a time constant known as the carrier lifetime, which greatly affects the performance of semiconductor devices during operation. As one of the important parameters for semiconductor devices and materials, the carrier lifetime is very sensitive to the presence of defects in these materials, and further requires a convenient method of evaluation. J. Warman and M. Kunst developed a transient technique they named the time resolved microwave conductivity (TRMC), which involves microwave absorption to follow charge carrier dynamics in semiconductors ${ }^{1}$. Other researchers proposed the transient photo conductivity (TPC), otherwise known as the microwave photoconductivity decay ( $\mu$-PCD), which is the commonly adopted material qualification technique in semiconductor industries due to its noncontact and nondestructive measurements of the carrier lifetime. In particular, for silicon carbide ( $\mathrm{SiC}$ ), three major techniques are applicable: $\mu-\mathrm{PCD}$, time resolved photoluminescence (TR-PL), and time resolved free carrier absorption (TR-FCA $)^{2,3,4,5,6,7}$. Among these techniques, $\mu-P C D$ is the most widely employed because compared to the other two as it exhibits surface roughness insensitivity (i.e., measurable for any given various surface roughness ${ }^{8,9,10}$ ) and high signal sensitivity for excited carriers (i.e., using an optimum microwave component). In general, $\mu-\mathrm{PCD}$ has been preferred for carrier lifetime measurement in SiC and other semiconductor materials $2,5,6,11,12,13,14,15,16,17,18,19$.

The measurement protocol and principle of $\mu-P C D^{1,20,21}$ is detailed here. In principle, it uses a reflected microwave as a probe. Here, the microwave reflectance of a sample $R(\sigma)$ is equivalent to the ratio between the reflected microwave intensity $P(\sigma)$ and the incident microwave intensity $P_{\text {in }}$ as expressed by Equation 1:

$R(\sigma)=\frac{P(\sigma)}{P_{\text {in }}}(1)$

By irradiation of a pulse laser, the conductivity of a sample $\sigma$ changes to $\sigma+\Delta \sigma$; likewise, $R(\sigma)$ transforms to $R(\sigma+\Delta \sigma)$. Thus, $\Delta R$ is given by Equation 2:

$\Delta R=R(\sigma+\Delta \sigma)-R(\sigma)=\frac{P(\sigma+\Delta \sigma)-P(\sigma)}{P_{\text {in }}}=\frac{\Delta P}{P_{\text {in }}}(2)$

In a perturbation (small $\Delta \sigma$ ) approximation, $R(\sigma+\Delta \sigma$ ) is developed in Taylor series to yield 
$\frac{\Delta P}{P_{\text {in }}}=\left[\frac{\delta R(\sigma)}{\delta \sigma}\right] \Delta \sigma,(3)$

while $\Delta \sigma$ becomes

$\Delta \sigma=q\left(\mu_{p}+\mu_{n}\right) \Delta p,(4)$

where $q$ is the elementary charge, $\mu_{p}$ is the hole mobility, $\mu_{n}$ is the electron mobility, and $\Delta p$ is the excess carrier concentration. From the preceding equations, $\Delta R$ and $\Delta p$ are related by

$\Delta R \propto q\left(\mu_{p}+\mu_{n}\right) \Delta p \cdot(5)$

The dependence of microwave reflectance on excess carrier concentration allows $\mu$-PCD to observe the time decay of excess carriers, which we can use to estimate the carrier lifetime of semiconductor materials.

\section{Protocol}

\section{Preparation of the sample}

1. Prepare an n-type $4 \mathrm{H}-\mathrm{SiC}$ epilayer (Table of Materials).

2. Wash the sample with acetone and then with water, each for $5 \mathrm{~min}$, using an

3. ultrasonic washer.

4. Use a nitrogen gun to remove moisture on the sample surface.

\section{Preparation of aqueous solutions}

1. Prepare $1 \mathrm{M}$ each of $\mathrm{H}_{2} \mathrm{SO}_{4}, \mathrm{HCl}, \mathrm{Na}_{2} \mathrm{SO}_{4}, \mathrm{NaOH}$, or $\mathrm{HF}$ at $1 \mathrm{wt} \%$ concentration. Select and prepare an aqueous solution to be measured.

2. Prepare a quartz cell with $5 \mathrm{~mm}$ (length) $\times 20 \mathrm{~mm}$ (width) $\times 40 \mathrm{~mm}$ (height) dimensions and then pour the aqueous solution into it. Put the prepared sample into the cell and then immerse it into the aqueous solution.

NOTE: At least $4 \mathrm{~mL}$ of the aqueous solution in the quartz cell is required for the sample to be immersed entirely. When changing the solution, treat the sample with ultrasonic cleaning using acetone and pure water.

\section{Preparation of the measuring equipment}

1. Turn on the power supply of the 266-nm pulsed laser to excite the light source. Afterwards, set the laser mode on standby.

2. Connect the pulsed laser and an oscillator through a Bayonet Neill-Concelman (BNC) cable. Turn on the oscillator and input a $100 \mathrm{~Hz}$ pulse wave to the pulsed laser.

3. Connect a photodiode for trigger acquisition through a trigger input channel of the oscilloscope with a BNC cable.

4. Turn on the power supply of the photodiode.

5. Irradiate the pulsed laser and place the aperture of the microwave waveguide on the optical path of the laser light at direction normal to the light.

CAUTION: In the latter process, the experimenter should wear safety eye glasses during laser irradiation.

6. Install a half-mirror on the optical path of the pulsed laser, as shown in Figure 1, and reflect the pulsed laser to the photodiode.

7. Turn on the oscilloscope and then set its trigger threshold to a voltage sufficient to signal from the photodiode. NOTE: The threshold value may be set smaller than the peak of the trigger signal. When an unintentional reflected light enters the photodiode, the oscilloscope displays a frequency that differs greatly from the pulsed laser frequency. In this case, repeat step 3.6.

8. Check the trigger frequency with an oscilloscope and tune the oscillator exactly.

9. Put the laser mode on standby.

10. Connect a Schottky barrier diode in a microwave waveguide for reflected microwave detection and a signal input channel of the oscilloscope, through a BNC cable.

11. Apply a $9.5 \mathrm{~V}$ operating voltage to a Gunn diode.

12. Place the quartz cell (step 2.2) on the stand in front of the aperture as close as possible. Fix with tape.

\section{Measurement and saving data}

1. Turn on the laser light oscillation and irradiate the light to the sample.

2. Place a half-waveplate $(\lambda / 2)$, a polarizer, and a power meter on the optical path (Figure 1).

3. Irradiate the pulsed laser to the power meter, as shown in Figure 1. Check the excitation intensity of the laser.

4. Adjust the $N / 2$ angle for control of the excitation intensity.

NOTE: $\lambda / 2$ changes the polarization direction of the laser light, while the polarizer transmits only one light polarization direction, through which excitation intensity is controlled. Injected photon densities are set to $8 \times 10^{13} \mathrm{~cm}^{-2}$ and for the 266-nm laser, the excitation carrier density in $4 \mathrm{H}-\mathrm{SiC}$ is $4.5 \times 10^{17} \mathrm{~cm}^{-3}$

5. Remove the power meter from the optical path.

6. Adjust the time/div and V/div of the oscilloscope so that the peak signal is displayed on the oscilloscope. 
7. Adjust the amplitude and phase of the microwave through an $\mathrm{E}-\mathrm{H}$ tuner. Check the oscilloscope and look for the $\mathrm{E}-\mathrm{H}$ tuner where the peak signal is at maximum. Failed adjustment of the $\mathrm{E}-\mathrm{H}$ tuner results in signal loss, as depicted in Figure 2.

NOTE: An amplifier is used to strengthen the decay signal in case of insufficiently large signal relative to background noise or when it is not observed even after adjusting the E-H tuner. The amplifier is placed between the Schottky barrier diode and the signal input channel of oscilloscope with a BNC cable, as illustrated in Figure 1.

8. Repeat steps 4.6 and 4.7 to complete the tuning.

9. Adjust the time/div of the oscilloscope and sketch a decay curve in the measurement area on the oscilloscope.

10. Average the signal for an arbitrary number of times to improve the signal-to-noise ratio.

11. Save the measurement data as an electronic file to a memory and then remove it from the oscilloscope.

\section{Data processing}

1. Import the signal data to a personal computer.

2. Plot decay curves obtained from the experiment as a function of time.

3. Calculate the average value of background noise level, subtract it from the decay signal, and plot it as a function of time.

4. Find the peak value of the decay signal obtained in step 5.3 and then divide the decay signal by the peak value.

\section{Representative Results}

Figure 1 shows a schematic diagram of the $\mu$-PCD apparatus consisting of a $10 \mathrm{GHz}$ microwave frequency, $\mathrm{X}$ waveguide band, and a rectangular waveguide. The microwave was focused by the double ridge waveguide and irradiated on the sample. The Gunn diode output power was $50 \mathrm{~mW}$ and the phase noise was nearly $-80 \mathrm{dBc} / \mathrm{Hz}$.

Figure 3 shows the $\mu$-PCD decay curve of a 100- $\mu$ m-thick n-type $4 \mathrm{H}-\mathrm{SiC}$ sample excited on the Si-face by $266 \mathrm{~nm}$ in the air; $\mu$-PCD signal (V) scaled logarithmically was the dependent variable and time $(\mu \mathrm{s})$ was the independent variable. The signal voltage peak was approximately $0.046 \mathrm{~V}$ prior to amplification. Moreover, the observed voltage of the direct current (DC) component of the reflected microwave obtained from the oscilloscope DC mode was of the order of several volts. As recombination of excess carriers progressed with time, the sample's conductivity and microwave reflectance decreased.

Figure 4 shows the normalized $\mu$-PCD decay curve of Figure 3. Normalization enables comparison of the time constants with different peak intensities. Typically, carrier lifetime estimation based on the decay curve is carried out with the 1/e lifetime $\tau_{1 / \mathrm{e}}$ parameter, indicating that time expended to obtain signal intensity decreases from the peak to $1 / \mathrm{e}(\sim 0.368)$. Note that $\mu$-PCD decay was not a single exponential and $\tau_{1 / \mathrm{e}}$ was influenced by both bulk and surface recombination. However, comparing the time constant of samples having different thickness or surface condition necessitated a reference parameter. Usage of $\tau_{1 / \mathrm{e}}$ was convenient given the good signal-to-noise ratio at the initial part of the decay curve and the simplicity of the data analysis. To characterize the $\mu$-PCD signal, half-time lives, $I_{40} / I_{\max }$, and $k_{\mathrm{D}}$ constant also adopted such parameters $22,23,24$. In fact, $\tau_{1 / \mathrm{e}}$ was adopted in the SEMI standard: SEMI MF $1535^{8}$ as the standard for carrier lifetime measurement of Si. For the decay curve in Figure 4, $\tau_{1 / \mathrm{e}}$ was approximately $0.34 \mu \mathrm{s}$.

In Figure 5, the quartz cell, containing the aqueous solution and with the sample on its wall, was placed on the stand in front of the aperture ${ }^{11}$. Each intensity of the irradiated microwave and the reflected microwave from the sample, as well as the $\mu$-PCD signal-to-noise ratio, were dependent on the distance between the sample and the aperture, which, ideally, should be as close as possible. In the actual measurement, the distance obtained was as close as possible; measurement using the quartz cell yielded a distance of $0.5 \mathrm{~mm}$, which was of the same as the thickness of the quartz cell glass.

Figure 6 shows $\mu$-PCD decay curves of the n-type $4 \mathrm{H}-\mathrm{SiC}$ in the air and in aqueous solutions. An excitation light of $266 \mathrm{~nm}$ was irradiated to

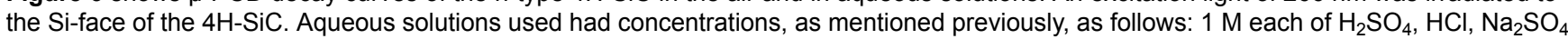
or $\mathrm{NaOH}$, or $1 \mathrm{wt} \%$ of HF. The time constant of the decay curves was longer with the sample immersed into the acidic aqueous solutions (i.e., $\mathrm{H}_{2} \mathrm{SO}_{4}, \mathrm{HCl}$, or $\mathrm{HF}$ ), implying that acidic solutions passivated surface states on the Si-face and reduced surface recombination of the excess carriers.

Figure 7 shows the $\mathrm{pH}$ dependence of $\tau_{1 / e}$ of the $\mathrm{n}$-type $4 \mathrm{H}$-SiC sample excited on the Si-face at $266 \mathrm{~nm}$ of light. The $\mathrm{pH}$ was calculated from the molar concentrations of $\mathrm{H}_{2} \mathrm{SO}_{4}, \mathrm{HCl}$, and $\mathrm{NaOH}$. This figure indicated the carrier lifetime dependence on pH aqueous solutions; therefore, lower $\mathrm{pH}$ would have more effects on the carrier lifetime.

Surface recombination velocity $S$ was calculated to reproduce the $\tau_{1 / \mathrm{e}}$ used for the samples. The decay model of excess carriers has been reported in refs. 2 and 3 . To obtain the excess carrier concentration $\operatorname{Dn}(x, t)$, the following continuity equation was solved. Here, $\mathrm{D} n(x, t)$ was defined as a function of time $t$ and depth $x$ in a semiconductor layer; thus,

$\frac{\partial \Delta n(x, t)}{\partial t}=D_{a} \frac{\partial^{2} \Delta n(x, t)}{\partial x^{2}}+\frac{\Delta n(x, t)}{\tau_{\mathrm{R}}}+B \Delta n^{2}+C \Delta n^{3}$,

where $T_{B}$ is bulk lifetime due to the Shockley-Read-Hall (SRH) recombination, $D_{\mathrm{a}}$ is the ambipolar diffusion coefficient, $B$ is the radiation recombination coefficient, and $C$ is the Auger recombination coefficient.

At the excited and other surfaces, boundary conditions were given by Equation 7:

$D_{a} \frac{\partial \Delta n(0, t)}{\partial x}=S_{0} \Delta n(0, t)$ and $D_{a} \frac{\partial \Delta n(W, t)}{\partial x}=S_{\mathrm{W}} \Delta n(W, t)$ 
where $S_{0}$ and $S_{W}$ denote the surface recombination velocity of the excited and other surfaces, respectively, and $W$ is the layer thickness.

Moreover, the initial excess carrier concentration profile using light pulse illumination could be expressed using Equation 8:

$\Delta n(x, 0)=g_{0} \exp (-a x)$

where $g_{0}$ is carrier concentration at $x=0$ and $a$ is the absorption coefficient.

Solving Equation 6 by employing the boundary conditions of Equation 7 and the initial condition of Equation 8 provided the excess carrier decay curves. In the process, $S$ was estimated by comparing the $\tau_{1 / \mathrm{e}}$ obtained from the experiments and from the calculated decay curves. Least squares fitting minimized errors between the experimental $\tau_{1 / \mathrm{e}}$ in the all conditions and the calculated $\tau_{1 / \mathrm{e}}$ with parameters $S_{0}, S_{\mathrm{w}}$, and $\tau_{\mathrm{B}}$.

As depicted in Equation 6 carrier recombination is the summation of various decay components, namely, surface, $\mathrm{SRH}$, radiative, and Auger recombinations, the last two having remarkable high carrier density. On the other hand, SRH recombination depends on point defects and dislocations in the bulk of the semiconductor material that form energy levels in the semiconductor band gap. The energy levels act as stepping stones for carrier transition between the valence and conduction bands.

$\mu$-PCD also shows nonlinearity at a high injection condition, and overestimates carrier lifetime ${ }^{13,25,26}$. Figure 8 shows the measured $\mu$-PCD under a high excitation condition. Note that the decay curve for an injected photon density of $10^{15} \mathrm{~cm}^{-2}$ became more gradual compared to that for photon density of $10^{14} \mathrm{~cm}^{-2}$, owing to microwave nonlinearity. Furthermore, the measurement examples shown in Figure 3, Figure 4, and Figure 6 were obtained for an injected photon density of $8 \times 10^{13} \mathrm{~cm}^{-2}$ resulting in negligible microwave nonlinearity, and Auger and radiative recombinations but dominant $\mathrm{SRH}$ and surface recombinations.

Figure 6 can be taken to exemplify decay curve calculations for the n-type $4 \mathrm{H}$-SiC Si-face excited by $266 \mathrm{~nm}$ light, by referring to the dashed lines, where $\tau_{\mathrm{B}}=3 \mu \mathrm{s}$ and $S$ for the Si-face $S_{\mathrm{Si}}=200 \mathrm{~cm} / \mathrm{s}$ or $700 \mathrm{~cm} / \mathrm{s}$. For both $S_{\mathrm{Si}}$ settings, the experimental decay curve measured in neutral $\mathrm{pH}\left(\right.$ air, $\left.1 \mathrm{M} \mathrm{Na}_{2} \mathrm{SO}_{4}\right)$ and in the acidic condition $\left(1 \mathrm{M} \mathrm{H}_{2} \mathrm{SO}_{4}\right)$, respectively, were well reproduced, which meant that $S_{\mathrm{Si}}$ for the $\mathrm{n}$-type $4 \mathrm{H}$-SiC significantly reduced from $700 \mathrm{~cm} / \mathrm{s}$ to $200 \mathrm{~cm} / \mathrm{s}$ in acidic aqueous solutions as hydrogen passivated the surface states on the Si-face.

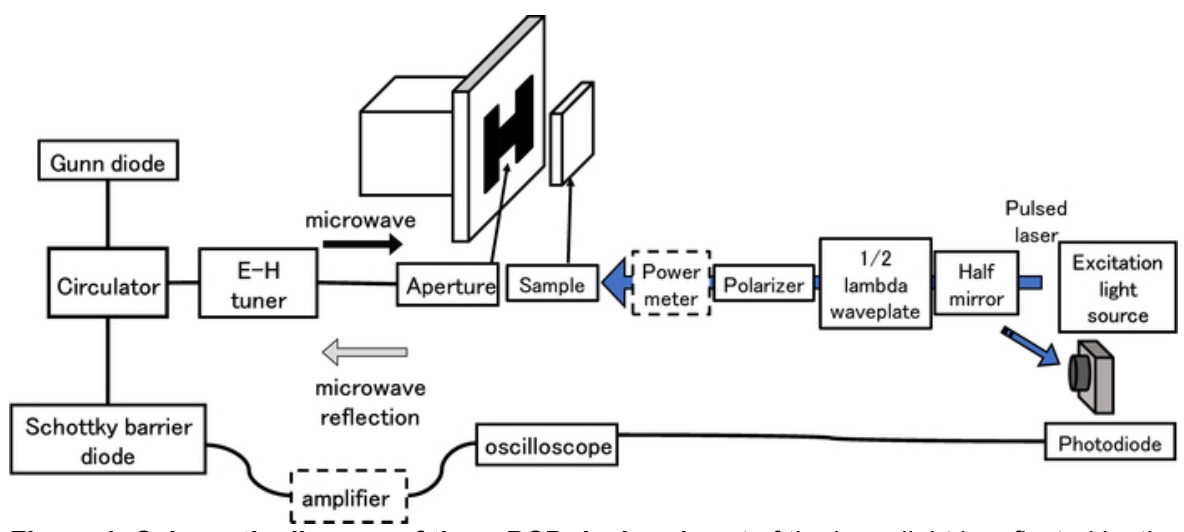

Figure 1: Schematic diagram of the $\mu-P C D$ device. A part of the laser light is reflected by the half-reflection mirror. The reflected laser is detected by the photodiode, and a signal coming from the photodiode is used as a trigger for the oscilloscope. A microwave is generated from the Gunn diode in the direction bent by the circulator; then, a microwave goes through the aperture and irradiates the sample. The reflected microwave from the sample comes back to the aperture and into the circulator, where it is detected by the Schottky barrier diode. Finally, the signal coming from the Schottky barrier diode is observed by the oscilloscope. Please click here to view a larger version of this figure. 


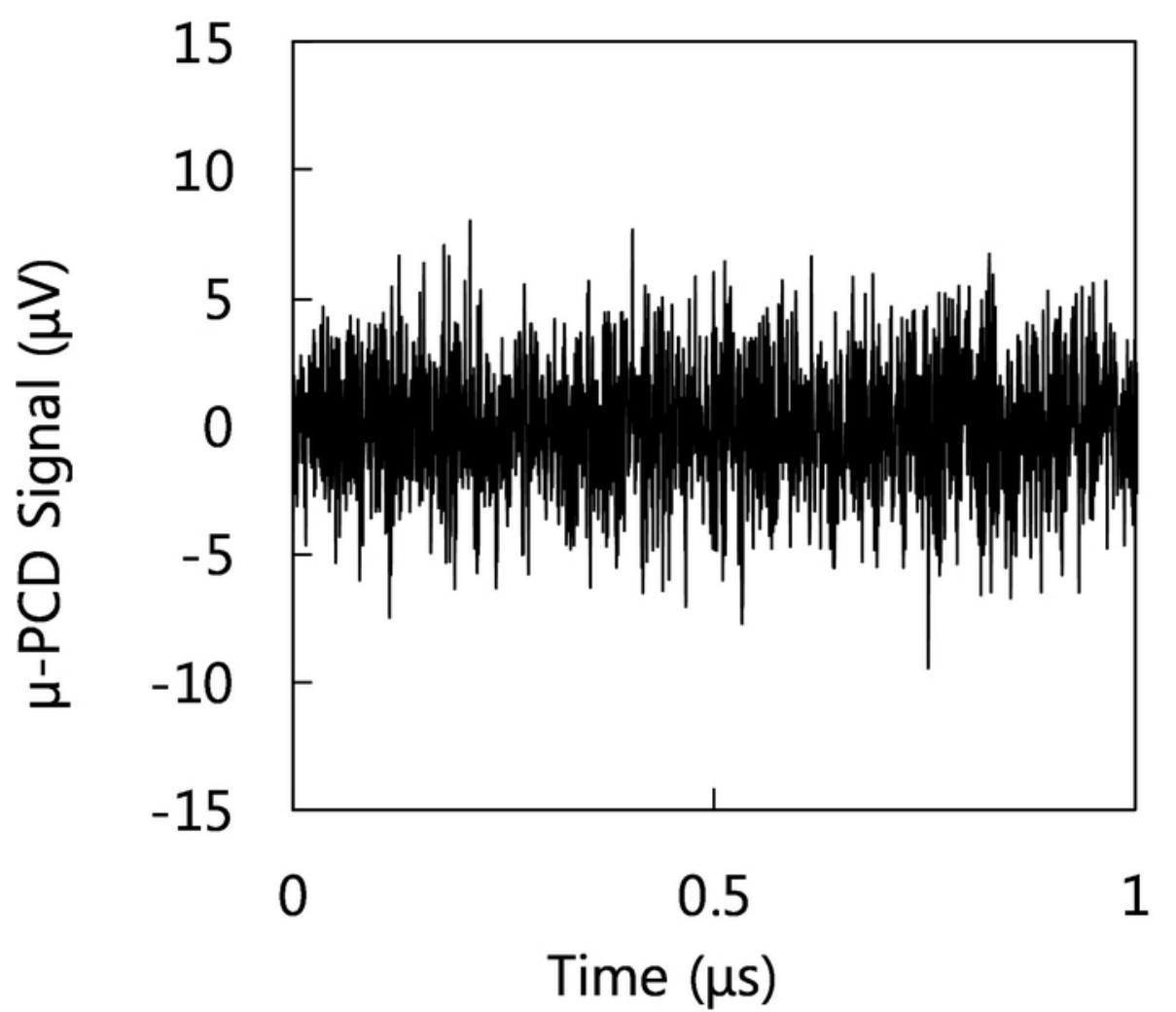

Figure 2: The $\mu-P C D$ signal for a failed tuning of E-H tuner. No measurable peak is observed for a failed tuning. Please click here to view a larger version of this figure.

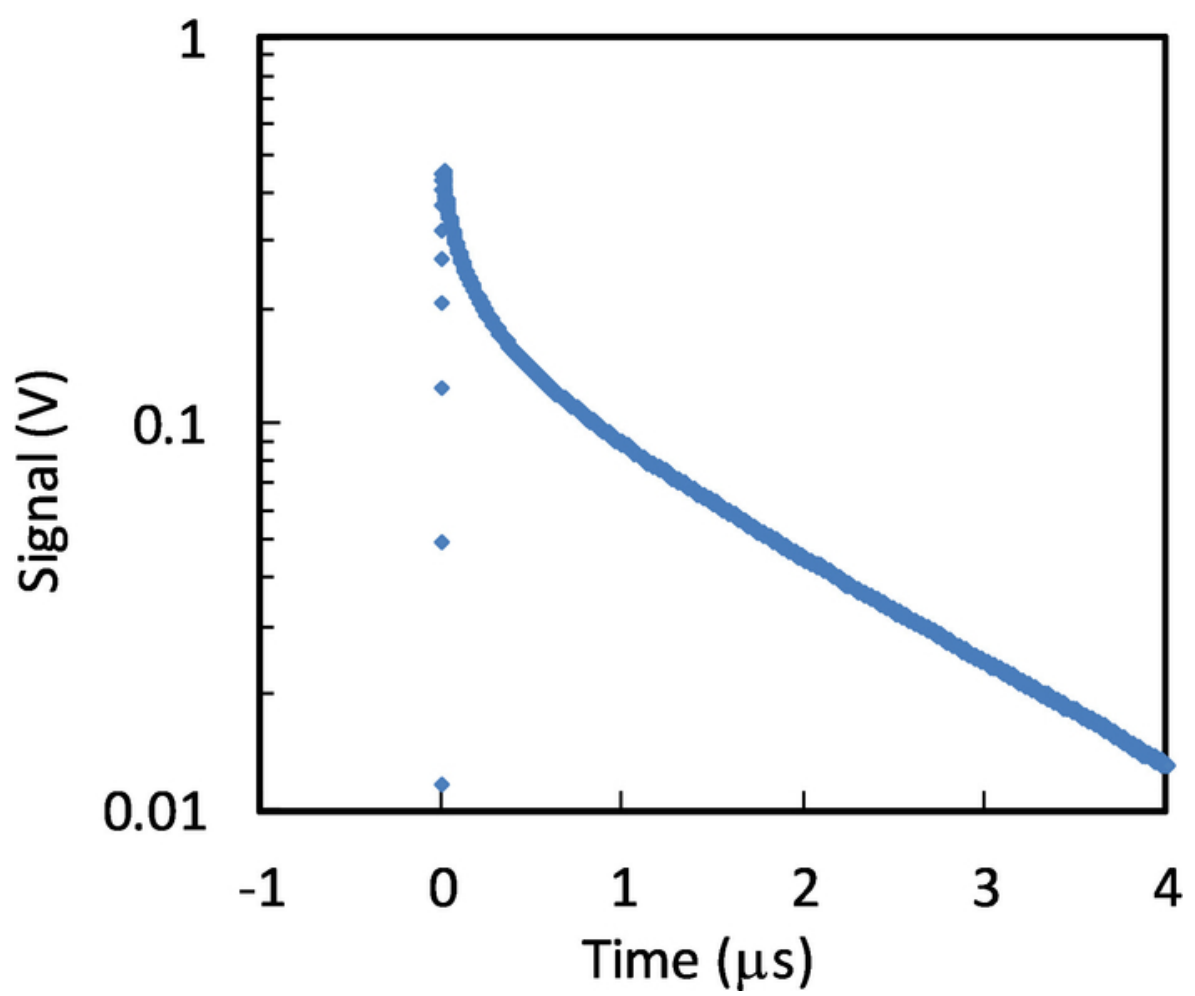

Figure 3: The $\mu$-PCD decay curve for the $\mathrm{n}$-type $4 \mathrm{H}-\mathrm{SiC}$ sample with excitation on the Si-face by $266 \mathrm{~nm}$ in air. The pulsed laser is irradiated at time $=0 \mathrm{~s}$ at which the signal intensity is at maximum. This figure has been modified from Ichikawa et al. ${ }^{11}$ with permissions. Please click here to view a larger version of this figure. 


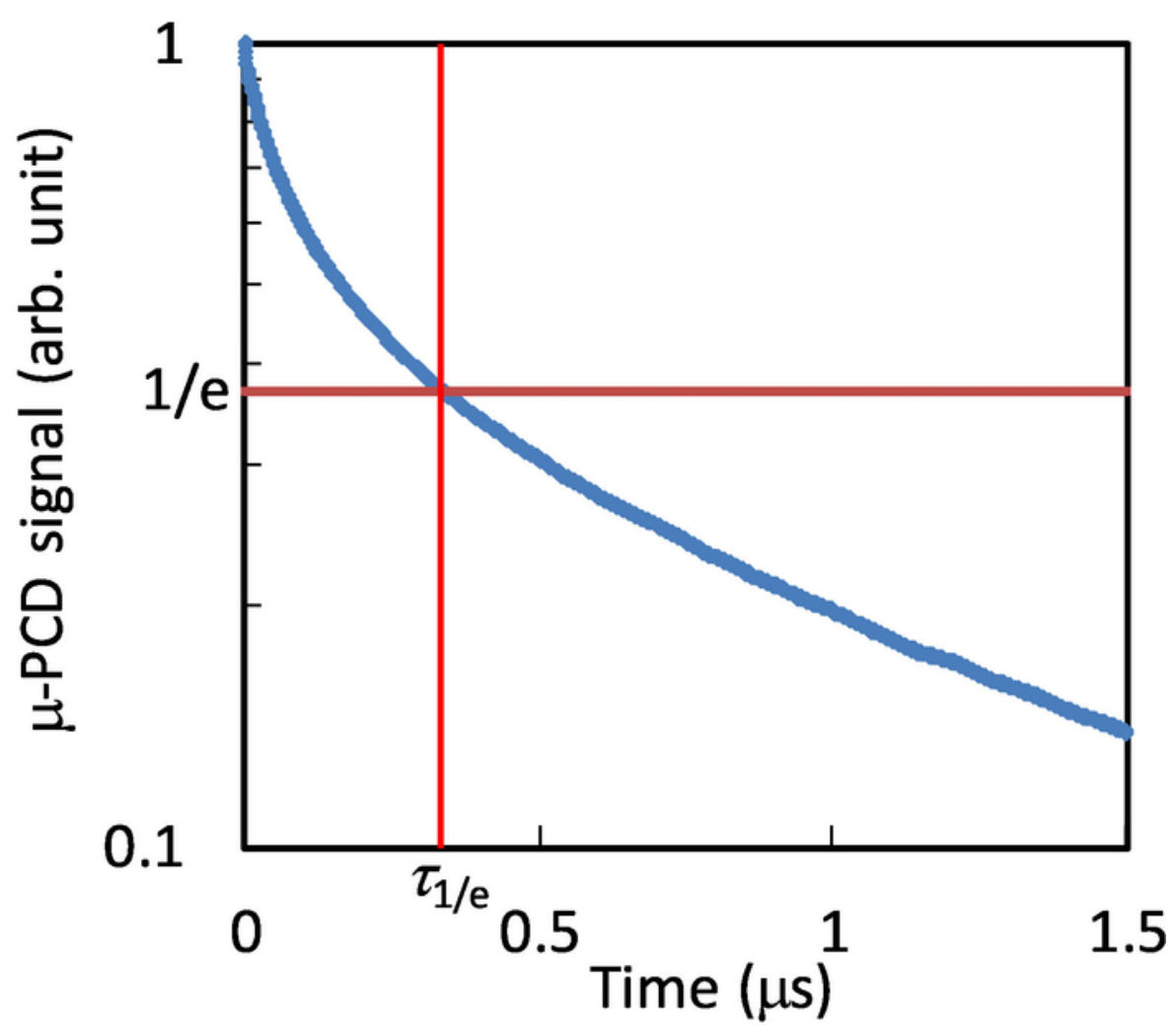

Figure 4: Normalized $\mu$-PCD decay curve for the $\mathrm{n}$-type 4H-SiC sample with excitation on the Si-face by $266 \mathrm{~nm}$ in air. The maximum value of the decay curve in Figure 2 is normalized to unity. The value of the dashed line is $1 / \mathrm{e}$, and $\tau_{1 / \mathrm{e}}$ is approximately $0.34 \mu \mathrm{s}$ as depicted. This figure has been modified from Ichikawa et al. ${ }^{11}$ with permissions. Please click here to view a larger version of this figure. 


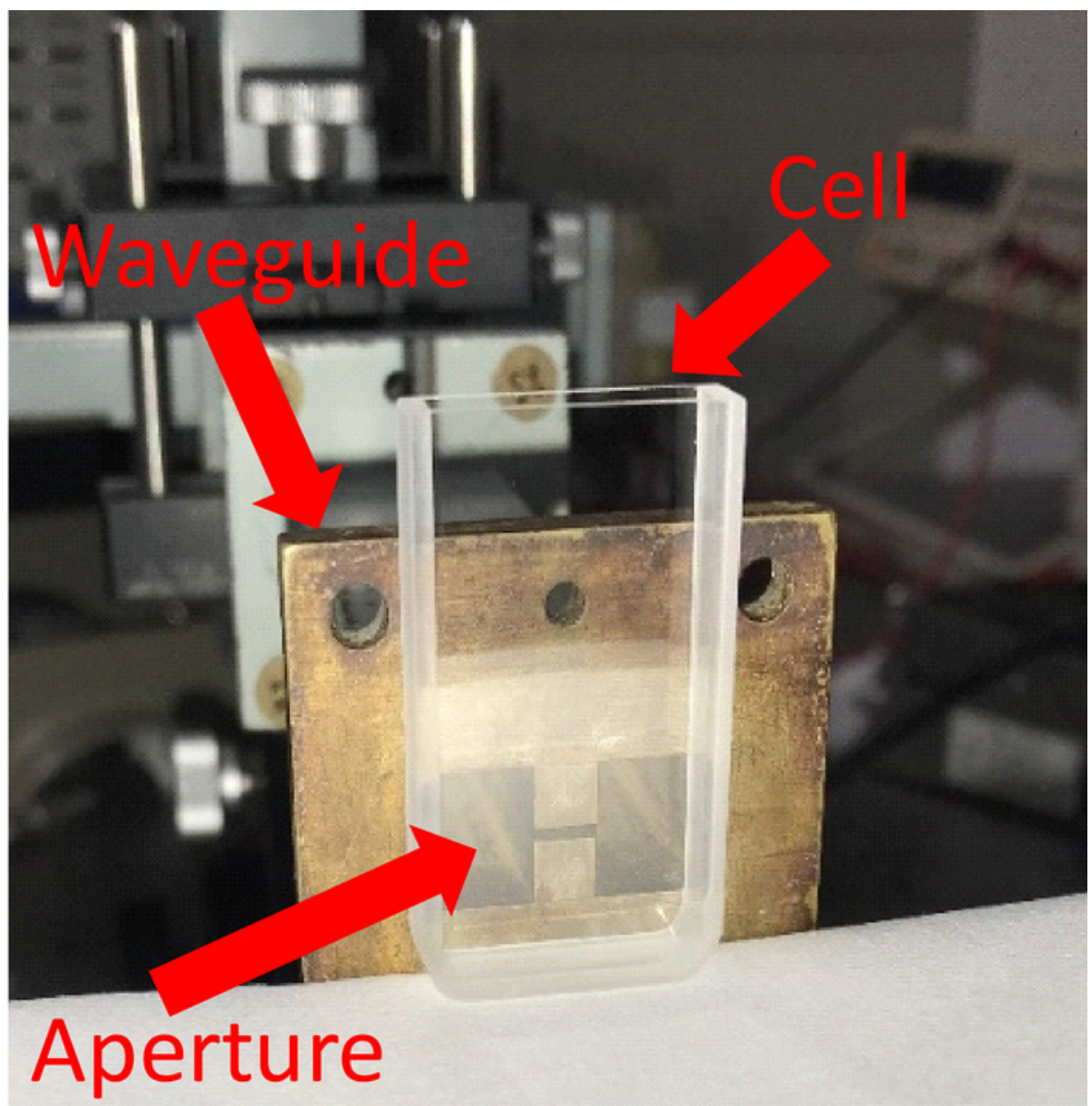

Figure 5: Image of $\mu$-PCD measurement in an aqueous solution in a quartz cell. The quartz cell is placed on the stand in front of the aperture to allow $\mu-P C D$ decay curve measurement in an aqueous solution. The cell dimension is $5 \mathrm{~mm} \times 20 \mathrm{~mm} \times 40 \mathrm{~mm}$ (length $\times$ width $\mathrm{x}$ height). Please click here to view a larger version of this figure. 


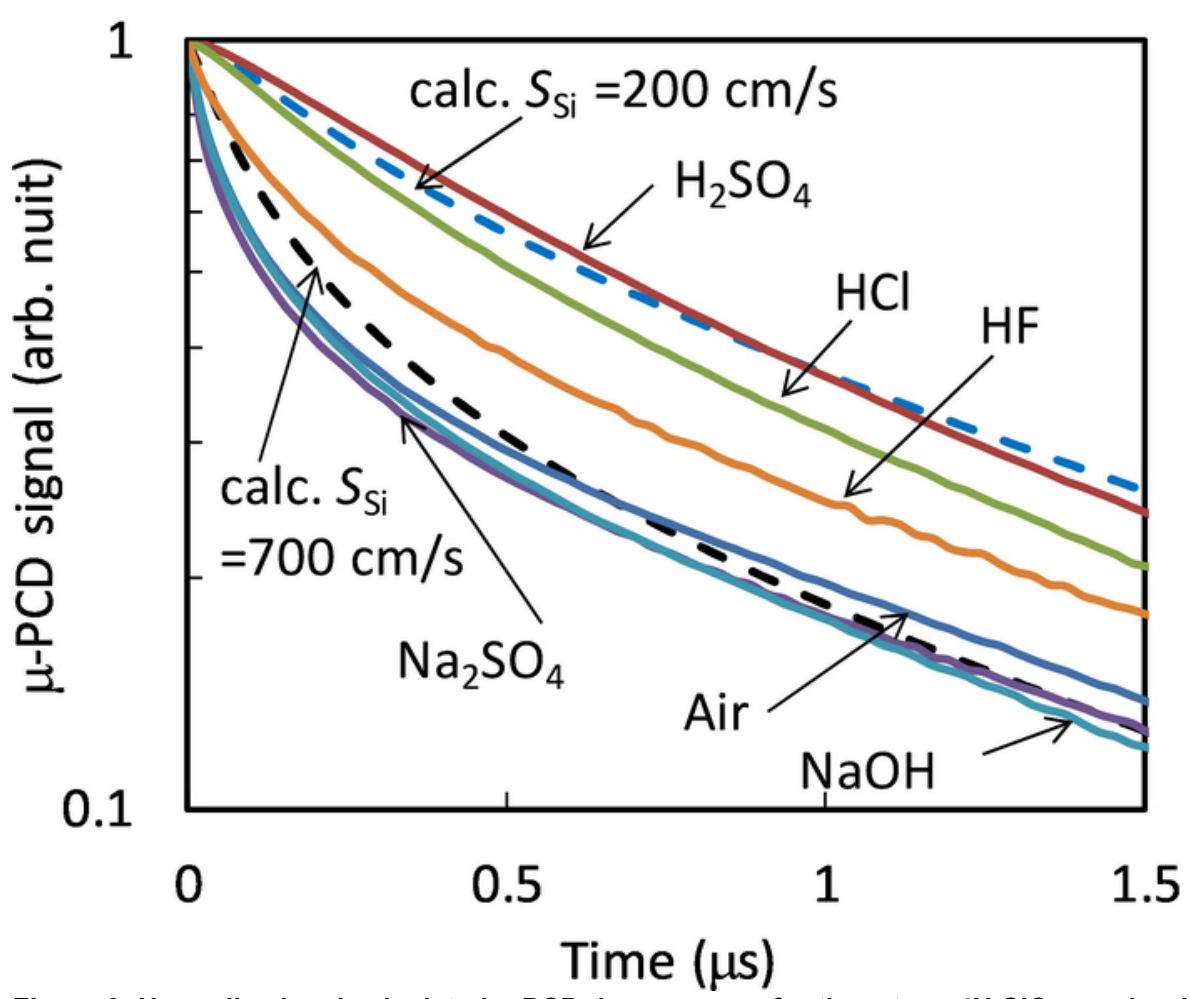

Figure 6: Normalized and calculated $\mu$-PCD decay curves for the n-type 4H-SiC sample with excitation on the Si-face by $266 \mathrm{~nm}$, in air and aqueous solutions. Solid lines represent the $\mu-\mathrm{PCD}$ experimental result curves for the aqueous solutions of $\mathrm{H}_{2} \mathrm{O}, \mathrm{H}_{2} \mathrm{SO}_{4}, \mathrm{HCL}_{2} \mathrm{Na}_{2} \mathrm{SO}_{4}$, $\mathrm{NaOH}$, or $\mathrm{HF}$. The dashed lines are calculated curves with the bulk carrier lifetime in the epilayers, $\tau_{\mathrm{B}}=3 \mu \mathrm{s}$, and the surface recombination velocity for the Si-face, $S_{\mathrm{Si}}=200 \mathrm{~cm} / \mathrm{s}$ or $700 \mathrm{~cm} / \mathrm{s}$. This figure has been modified from Ichikawa et al. ${ }^{11}$ with permissions. Please click here to view a larger version of this figure.

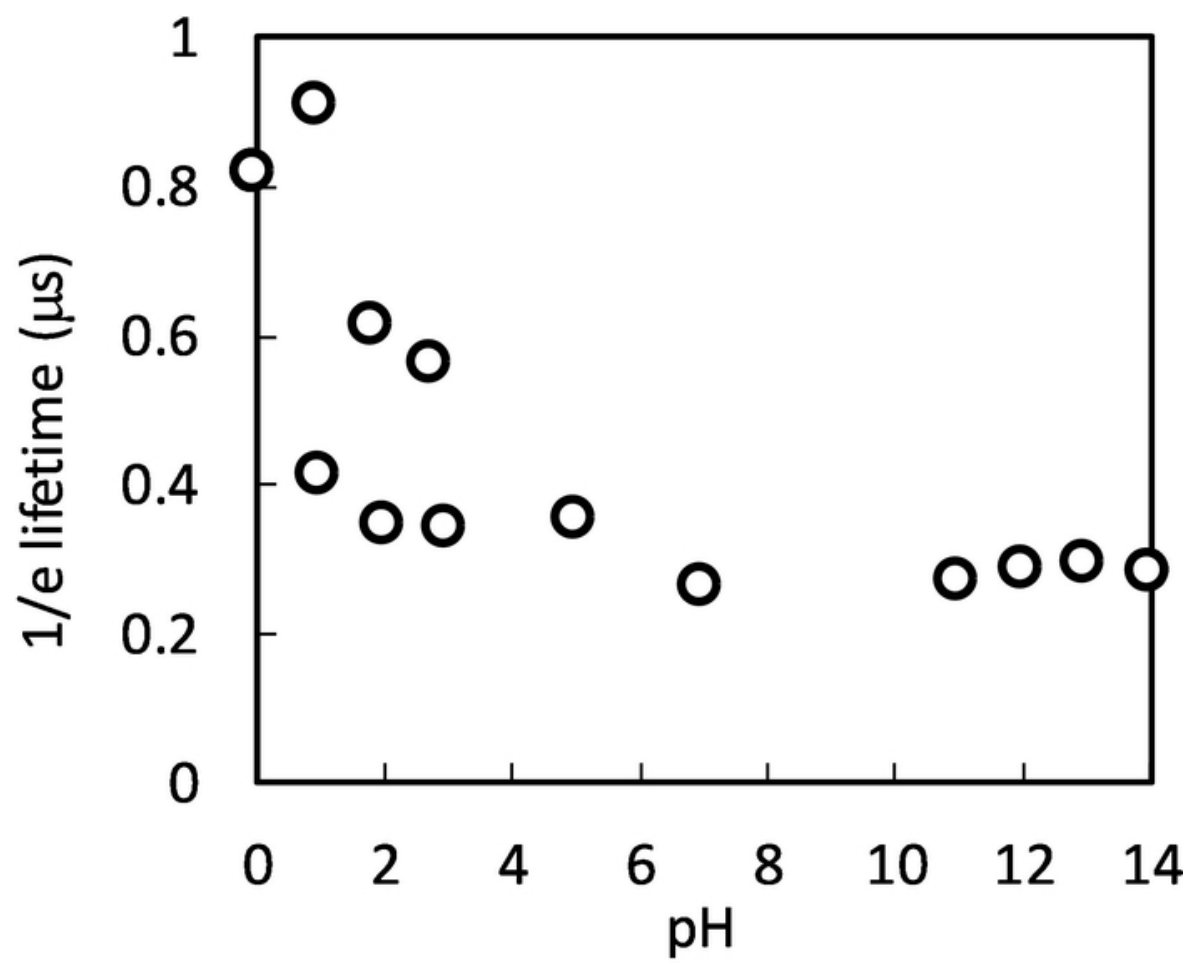

Figure 7: The $\mathrm{pH}$ dependence of $\boldsymbol{\tau}_{1 / \mathrm{e}}$ for the $\mathrm{n}$-type $\mathbf{4 H}$-SiC sample with excitation on the Si-face by $\mathbf{2 6 6} \mathbf{n m}$. Carrier lifetime increases as the $\mathrm{pH}$ of the aqueous solution decreases. This result indicates that lower $\mathrm{pH}$ will have more effects on the carrier lifetime. This figure has been modified from Ichikawa et al. ${ }^{11}$ with permissions. Please click here to view a larger version of this figure. 


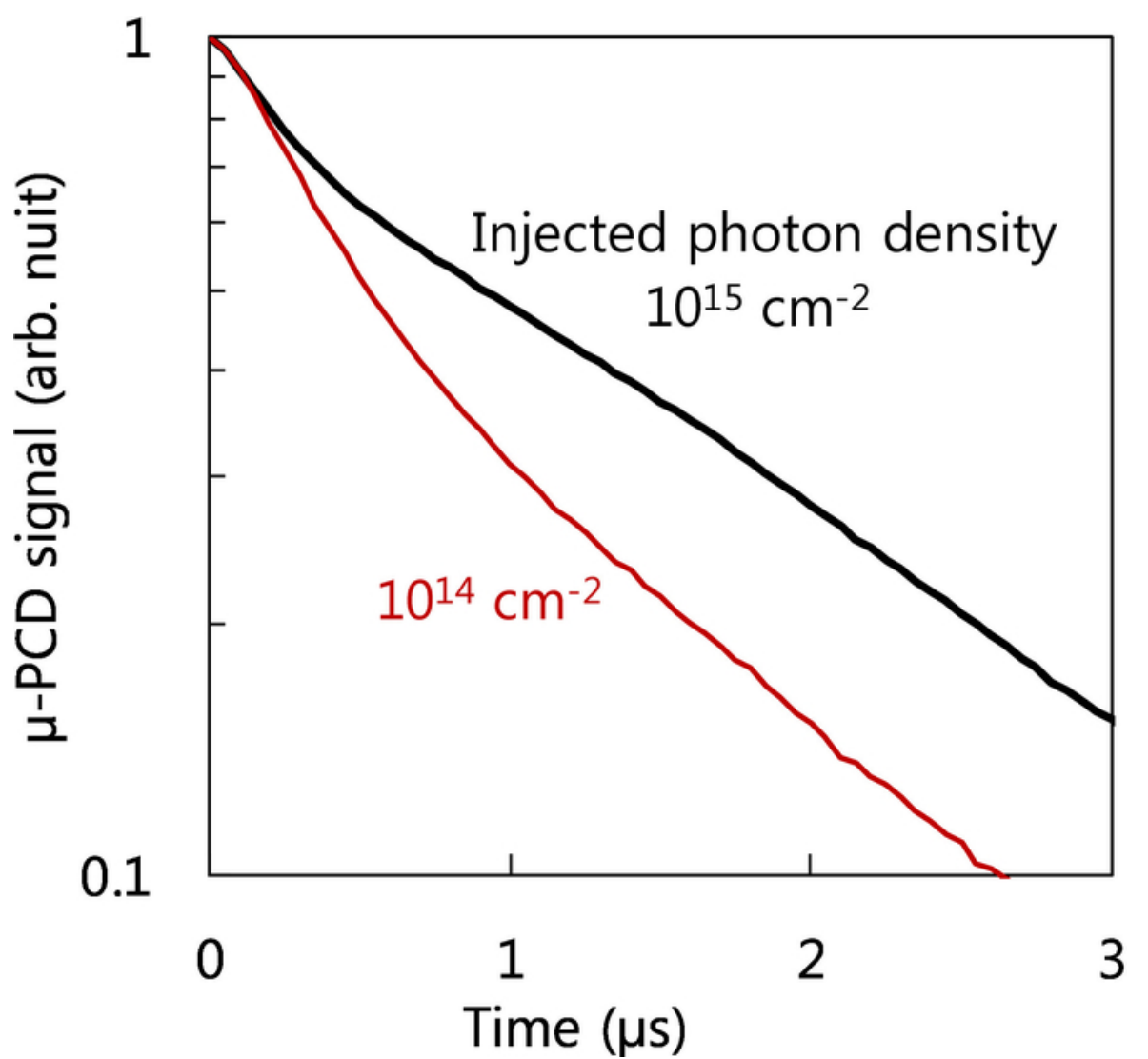

Figure 8: The $\mu-P C D$ decay curve of n-type 4H-SiC with excitation of injected photon density of $10^{14}$ or $10^{15} \mathrm{~cm}^{-2}$ on the Si-face by 266 $\mathrm{nm}$. Measurement with high excitation at photon density of $10^{15} \mathrm{~cm}^{-2}$ makes a more gradual decay curve than that with lower photon density due to nonlinearity of microwave reflectivity. Please click here to view a larger version of this figure.

\section{Discussion}

In the $\mu$-PCD protocol, step 4.7 is the most important point. The $\mathrm{E}-\mathrm{H}$ tuner was incorporated with a movable short circuit in the $\mathrm{E}$ and $\mathrm{H}$ planes, respectively. Thus, moving the short circuit of the $\mathrm{E}$ tuner or the $\mathrm{H}$ tuner changes the amplitude and phase of the reflected microwave and maximizes the signal amplitude. Tuning has a large influence on the waveform of the decay curve and must be performed strictly. In case of a weak signal strength where tuning might be difficult, a few tens of tuning averages may be used. For failed tuning, the $\mu$-PCD decay curves are not observable; only the noise signal of an oscilloscope is observed. Figure $\mathbf{2}$ shows the oscilloscope waveform in such a case.

It is easy to measure highly resistive samples as there is no lower conductivity limit. When the sample resistivity is low or when the sample is thick, the skin effect of the microwave is not negligible. The distance until the electric field intensity of the microwave becomes $1 / \mathrm{e}$ times is referred to as skin depth $\delta$, which is expressed by Equation 9:

$\delta=\left[\frac{\omega \mu}{2}\left(\sqrt{(\omega \varepsilon)^{2}+\rho^{-2}}\right)-\omega \varepsilon\right]^{-\frac{1}{2}}$

where $\omega$ is the angular frequency of the microwave, and $\varepsilon, \rho$, and $\mu$ represent the sample's dielectric constant, resistivity, and permeability, respectively. In the case of $\mathrm{Si}$ and $\mathrm{SiC}$, approximate $\delta$ values for the $10 \mathrm{GHz}$ microwave were $9 \mathrm{~mm}$ at $50 \Omega \cdot \mathrm{cm}, 2 \mathrm{~mm}$ at $10 \Omega \cdot \mathrm{cm}, 500 \mu \mathrm{m}$ at $1 \Omega \cdot \mathrm{cm}$, and $150 \mu \mathrm{m}$ at $0.1 \Omega \cdot \mathrm{cm}$. Therefore, measurements for samples with typical thicknesses (several hundred micron) at less than 0.1 $\Omega \cdot \mathrm{cm}$ will lose $\delta$ accuracy. On the other hand, the microwave and optical radiation are incident from the opposite of the wafer in this protocol. A negligible skin effect indicates better microwave and optical radiation from the same side.

Lower limits depend on the resistivity and thickness of the sample resulting from its interaction with the microwave. For highly resistive samples, the typical lower limits of the excess carriers are on the order of $10^{12} \mathrm{~cm}^{-3}$. On the other hand, electron-hole scattering must be considered at excess carriers greater than $10^{16} \mathrm{~cm}^{-3}$, as discussed in ref. 13 .

The $\mu-P C D$ decay curves became gentle at high excitation density due to unproportionality of the microwave reflectivity to the excess carrier concentration such that Equation (3) would lose its validity ${ }^{13,25,26}$ and $\tau_{1 / \mathrm{e}}$ would be overestimated. Figure 8 shows the $\mu$-PCD decay curve of a chemical mechanical polishing surface treatment $\mathrm{n}$-type $4 \mathrm{H}-\mathrm{SiC}$ with excitation on the Si-face by $266 \mathrm{~nm}$ under high excitation intensity. 
Moreover, time resolution depends on the performance of the measurement apparatus such as an excitation source, an oscilloscope, and an amplifier. For example, in this study, the apparatus consisted of a pulsed laser with pulse width of $1 \mathrm{~ns}$ as the excitation source and an oscilloscope having a frequency band of $500 \mathrm{MHz}$. Consequently, the minimum measurable lifetime was estimated at $2 \mathrm{~ns}$.

As mentioned earlier, $\mu$-PCD is very useful for characterization of semiconductors such as Si. Nevertheless, its application can be extended to other materials, for instance, in photoactive materials including $\mathrm{TiO}_{2}{ }^{27,28,29,30}$.

Furthermore, aside from the $\mu-P C D, T R-P^{2}$ and TR-FCA introduced at the earlier sections are the other two carrier lifetime measurement techniques. TR-PL observes the time change of photoluminescence caused by carrier recombination while TR-FCA observes the time change of probe light absorption ${ }^{4}$. Specifically, free carrier absorption occurs when light with energy smaller than the band gap is irradiated during carrier excitation ${ }^{3}$. Nevertheless, compared to these two, $\mu-P C D$ directly observes electrical conductivity by microwave and has a high surface roughness and signal sensitivity, making it the more ideal method for carrier lifetime measurement for semiconductor device applications.

\section{Disclosures}

The authors have nothing to disclose.

\section{Acknowledgments}

This work was supported by the Nagoya Institute of Technology, Japan.

\section{References}

1. Kunst, M., Beck, G. The study of charge carrier kinetics in semiconductors by microwave conductivity measurements. Journal of Applied Physics. 60 (10), 3558-3566 (1986).

2. Klein, P. B. Carrier lifetime measurement in n-4H-SiC epilayers. Journal of Applied Physics. 103, 033702 (2008).

3. Linnros, J. Carrier lifetime measurements using free carrier absorption transients. I. Principle and injection dependence. Journal of Applied Physics. 84 (1), 275-283 (1998).

4. Mae, S., Tawara, T., Tsuchida, H., Kato, M. Microscopic FCA System for Depth-Resolved Carrier Lifetime Measurement in SiC. Materials Science Forum. 924, 269-272 (2018).

5. Miyazawa, T., Ito, M., Tsuchida, H. Evaluation of long carrier lifetimes in thick $4 \mathrm{H}$ silicon carbide epitaxial layers. Applied Physics Letters. 97 , 202106 (2010)

6. Kimoto, T., Danno, K., Suda, J. Lifetime-killing defects in $4 \mathrm{H}-\mathrm{SiC}$ epilayers and lifetime control by low-energy electron irradiation. Physica Status Solidi B. 245, 1327 (2008).

7. Ščajev, P., Gudelis, V., Jarašiūnas, K., Klein, P. B. Fast and slow carrier recombination transients in highly excited $4 \mathrm{H}$-and $3 \mathrm{C}$-SiC crystals at room temperature. Journal of Applied Physics. 108, 023705 (2010).

8. SEMI Standard. SEMI MF1535. (2007).

9. Hashizume, H., Sumie, S., Nakai, Y. Carrier Lifetime Measurements by Microwave Photoconductivity Decay Method. ASTM Special Technical Publication. 1340, 47 (1998).

10. Schöfthaler, M., Brendel, R. Sensitivity and transient response of microwave reflection measurements. Journal of Applied Physics. 77, 3162 (1995).

11. Ichikawa, Y., Ichimura, M., Kimoto, T., Kato, M. Passivation of Surface Recombination at the Si-Face of $4 \mathrm{H}-\mathrm{SiC}$ by Acidic Solutions. ECS Journal Solid State Science and Technology. 7 (8), Q127-Q130 (2018).

12. Mori, Y., Kato, M., Ichimura, M. Surface recombination velocities for $\mathrm{n}$-type $4 \mathrm{H}-\mathrm{SiC}$ treated by various processes. Journal of Physics $D$ : Applied Physics. 47, 335102 (2014).

13. Kato, M., Mori, Y., Ichimura, M. Microwave reflectivity from $4 \mathrm{H}-\mathrm{SiC}$ under a high injection condition: impacts of electron-hole scattering. Journal of Applied Physics. 54, 04DP14 (2015).

14. Kato, M., Matsushita, Y., Ichimura, M., Hatayama, T., Ohshima, T. Excess Carrier Lifetime in p-Type 4H-SiC Epilayers with and without LowEnergy Electron Irradiation. Japanese Journal of Applied Physics. 51, 028006 (2012).

15. Kato, M., Yoshida, A., Ichimura, M. Estimation of Surface Recombination Velocity from Thickness Dependence of Carrier Lifetime in n-Type 4H-SiC Epilayers. Japanese Journal of Applied Physics. 51, 02BP12 (2012).

16. Mori, T. et al. Excess Carrier Lifetime Measurement of Bulk SiC Wafers and Its Relationship with Structural Defect Distribution. Japanese Journal of Applied Physics. 44, 8333 (2005).

17. Jenny, J. R. et al. Effects of annealing on carrier lifetime in 4H-SiC. Journal of Applied Physics. 100, 113710 (2006).

18. Hayashi, T., Asano, K., Suda, J., Kimoto, T., Temperature and injection level dependencies and impact of thermal oxidation on carrier lifetimes in p-type and n-type 4H-SiC epilayers. Journal of Applied Physics. 109, 014505 (2011).

19. Okuda, T., Miyake, H., Kimoto, T., Suda, J. Long Photoconductivity Decay Characteristics in p-Type 4H-SiC Bulk Crystals. Japanese Journal of Applied Physics. 52, 010202 (2013).

20. Schofthaler, M., Brendel, R. Sensitivity and transient response of microwave reflection measurements. Journal of Applied Physics. 77, 3162-3173 (1995).

21. Beck, G., Kunst, M. Contactless scanner for photoactive materials using laser-induced microwave absorption. Review of Scientific Instruments. 57, 2 197-201 (1986).

22. Kolen'ko, Y. V, Churagulov, B. R., Kunst, M., Mazerolles, L., Colbeau-Justin, C. Photocatalytic properties of titania powders prepared by hydrothermal method. Applied Catalysis B: Environmental. 54, 51-58 (2004).

23. Carneiro, J. T., Savenije, T. J., Moulijn, J. A., Mul, G. The effect of Au on TiO2 catalyzed selective photocatalytic oxidation of cyclohexane. Journal of Photochemistry and Photobiology A: Chemistry. 217, 326-332 (2011). 
24. Luna, A. L. et al. Synergetic effect of Ni and Au nanoparticles synthesized on titania particles for efficient photocatalytic hydrogen production. Applied Catalysis B: Environmental. 191, 18-28 (2016).

25. Kunst, M., Beck, G. The study of charge carrier kinetics in semiconductors by microwave conductivity measurements. Journal of Applied Physics. 60, 3358 (1986).

26. Kunst, M., Beck, G. The study of charge carrier kinetics in semiconductors by microwave conductivity measurements. II. Journal of Applied Physics. 63, 1093 (1988).

27. Schindler, K.-M., Kunst, M. Charge-Carrier Dynamics in $\mathrm{TiO}_{2}$ Powders. The Journal of Physical Chemistry. 94, 8222-8226 (1990).

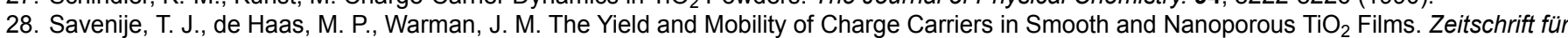
Physikalische Chemie. 212, 201-206 (1999).

29. Colbeau-Justin, C., Kunst, M., and Huguenin, D. Structural influence on charge-carrier lifetimes in $\mathrm{TiO}_{2}$ powders studied by microwave absorption. Journal of Materials Science. 38, 2429-2437 (2003).

30. Kato, M., Kohama, K., Ichikawa, Y., Ichimura, M., Carrier lifetime measurements on various crystal faces of rutile $\mathrm{TiO}_{2}$ single crystals. Materials Letters. 160, 397-399 (2015). 